\title{
A Study of the impact of Eyelid Opening and Closing on the Volume and Morphology of the Lacrimal Sac
}

\author{
Tu Yunhai, Wu W*, Chen $\mathbf{Y}$ and Shi J
}

Eye Hospital of Wenzhou Medical University, Wenzhou City, China

*Corresponding author: Wu W, Eye Hospital of Wenzhou Medical University, Wenzhou City, China, E-mail: wuwencan118@163.com

Received date: March 12, 2018; Accepted date: March 23, 2018; Published date: March 30, 2018

Copyright: $\odot 2018$ Wu W, et al. This is an open-access article distributed under the terms of the Creative Commons Attribution License, which permits unrestricted use, distribution, and reproduction in any medium, provided the original author and source are credited.

\begin{abstract}
Purpose: To study the impact of eyelid opening and closing on the volume and morphology of the lacrimal sac using computed tomography (CT) dacryocystography.

Method: All of the patients underwent CT dacryocystography. After the contrast agents was injected into a patient's lacrimal sac, the orbit was first scanned with the eye open. And then the patient was told to close the eye firmly for a second scan of the orbit. The volume of the lacrimal sac was calculated by the image processing program which came with the CT machine. At the same time, the anteroposterior and transverse diameters of the lacrimal sac at $3 \mathrm{~mm}$ and $7 \mathrm{~mm}$ from the top of the lacrimal sac were measured.

Results: 14 patients (14 eyes) who met the inclusion criteria were collected between December 2015 and January 2016. The average volume of the lacrimal sac obtained from the calculation based on CT dacryocystography was $0.165 \pm 0.073 \mathrm{~cm}^{2}$ when the eyelid was open and $0.165 \pm 0.076 \mathrm{~cm}^{2}$ when the eyelid was closed, there is no statistical difference. When the eyelid was open or closed, except for the anteroposterior diameter at the upper portion of the lacrimal sac, the differences in the transverse and anteroposterior diameters at the two positions of the lacrimal sac were no statistical difference.

Conclusion: When the eyelid is opened or closed, there is no significant change in the lacrimal sac volume or morphology.
\end{abstract}

Keywords: Lacrimal sac; Dacryocystography; Tear drainage; Mechanism

\section{Introduction}

Studying the mechanism of tear drainage is important for understanding and solving tearing in patients; however, the mechanism of tear drainage is currently unclear. Current theoretical hypothesis includes the lacrimal pump mechanism, a canalicular pump mechanism, and a tricompartment model of the lacrimal pump mechanism. The lacrimal pump mechanism proposed by Jones et al. [1] is mainly based on the anatomical structure of the lacrimal duct. The theory suggests that the orbicularis oculi muscle contracts when the eyelid is closed, thereby enabling lacrimal sac expansion (a volume increase) to create negative pressure in the lacrimal sac, encouraging tears to enter. When the eyelid is opened, due to elastic resilience, the lacrimal sac returns to its original volume (a volume decrease), creating positive pressure that prompts tears to enter the nasal cavity The canalicular pump mechanism proposed by Doane et al. [2] suggests that when the eyelid is closed, the canaliculus contracts, and the lacrimal punctum is closed, and the tears are squeezed into the lacrimal sac. When the eyelid is opened, the canaliculus expands, and the tears are sucked in, although the lacrimal sac volume does not change. Bercker et al. [3] proposed a "tricompartment model of the lacrimal pump mechanism" that suggests that when the eyelid is closed, the orbicularis oculi muscle contracts to compress the canaliculus and drag the upper portion of the outer wall of the lacrimal sac outward so that the upper portion of the lacrimal sac creates a lower pressure, prompting the tears to enter the lacrimal sac through the canaliculus. Meanwhile, the lower portion of the lacrimal sac wall is pulled inward so that positive pressure is created in the lower portion of the lacrimal sac and nasolacrimal duct, encouraging the lacrimal fluid to run down to be excreted into the nasal cavity. When the eyelid is opened, the muscles are relaxed, the canaliculus returns to its original state, and the upper portion of the outer wall of the lacrimal sac moves inward so that negative pressure is created in the canaliculus. When the lacrimal fluid flows into the canaliculus from the lacrimal lake, the interior pressure in the upper portion of lacrimal sac increases so that the Rosenmuller valve is closed. At the same time, the lower portion of the outer wall of the lacrimal sac moves outward so that the lower lacrimal sac and the nasolacrimal duct create negative pressure to push the lacrimal fluid to flow into their lower portions. Therefore, an important difference among the above-mentioned theories lies in the changes in the lacrimal sac volume and morphology when the eyelid is opened and closed.

Previously, other researchers also studied the change in the lacrimal sac volume when the eyelid was opened or closed; however, their study results had obvious contradictions. Amrith et al. [4] used magnetic resonance imaging (MRI) dacryocystography to study the change in the lacrimal sac volume when the eyelid was opened or closed, and the result showed that the lacrimal sac volume did not significantly change. However, the lacrimal sac morphology was not analyzed. While using fluoroscopic dacryocystography, Lee et al. [5] found that when the eyelid was closed, the width of the upper portion of the lacrimal sac increased, while the width of the lower portion of the 
Citation: Wu W, Tu Yunhai.Thinks, Chen Y, Shi J (2018) A Study of the impact of Eyelid Opening and Closing on the Volume and Morphology of the Lacrimal Sac. J Eye Dis Disord 3: 116.

Page 2 of 6

lacrimal sac did not significantly change. However, they did not show data on the lacrimal sac volume. Using ultrasound scanning technology, Pavlidis et al. [6] found that the lacrimal sac of volunteers with an unobstructed lacrimal duct was compressed by $50 \%$ when the eyelid was closed, while the compression was only $15 \%$ in volunteers with an obstructed lacrimal duct. However, B ultrasound examination may press on the lacrimal sac, thus interfering with the examination results.

Therefore, this study used a lacrimal sac computed tomography (CT) dacryocystography technique to measure the changes in the lacrimal sac volume and the morphology of the upper and lower portions of the lacrimal sac when the eyelid was opened or closed in patients with an obstructed nasolacrimal duct to investigate the role of the lacrimal sac in the mechanism of lacrimal fluid drainage.

\section{Methods}

\section{General information}

Patients who were diagnosed with chronic dacryocystitis in a single eye at the Eye Hospital of Wenzhou Medical University between September 2015 and January 2016 were enrolled. Patients with a small lacrimal sac and blockage of the inferior lacrimal duct were excluded. Patients with previous eye/nose surgery, an injury history, a history of acute dacryocystitis or obvious scarring or inner canthus deformity were also excluded. All of the patients signed an informed consent form, and the study was approved by the ethical review board for human testing.

\section{CT dacryocystography examination}

A CT machine (SOMATOM Emotion) with a 16-slice configuration was used. Before the operation, a $0.9 \%$ sodium chloride injection was used to wash the patient's lacrimal duct, and it was then ensured that the flush fluid did not contain secretions. The patient was placed in the supine position. An iopromide injection (approximately $0.5 \mathrm{ml}$ for each eye, with an iodine concentration of $300 \mathrm{mg} / \mathrm{ml}$ ) was drawn into a syringe, and a lacrimal cannula was then attached to the syringe to slowly inject the contrast agent via the inferior punctum. Each patient was asked to open his/her eyes, and the orbit was scanned by CT (thinlayer scanning in the sagittal position, coronal position and axial position, with a scanning time of $20 \mathrm{~s}$ ). The window width was 1500 $\mathrm{HU}$, the window level was $450 \mathrm{HU}$, and the layer thickness was 0.75 $\mathrm{mm}$. After the images were saved, the patient was asked to close his/her eyes firmly. The orbit was then scanned using the same settings as in the open-eye case, and the images were saved. All of the above CT scans were completed by a clinically experienced physician from the imaging department. Patients with clear dacryocystography images were included in the current study.

\section{Measurement of the lacrimal sac volume and cross-section}

The volume calculation program (syngo) in the image processing system that came with the CT machine was used to rebuild each image obtained from scanning in the axial position according to the scanlayer thickness of $0.75 \mathrm{~mm}$. Each region of the lacrimal sac that was filled with the contrast agent was assessed to obtain the area, which was multiplied by $0.75 \mathrm{~mm}$ to obtain the volume of this portion. The volumes of all of the lacrimal sac portions that were visualized using the contrast agent were obtained in this way, and their sum was the total volume of the lacrimal sac. Additionally, the horizontal section at $3 \mathrm{~mm}$ from the top of the lacrimal sac was used to study the morphology of the upper portion of the lacrimal sac, and the horizontal section at $7 \mathrm{~mm}$ from the top was used to study the morphology of the lower portion of the lacrimal sac. The built-in measurement function of the CT system could be used to directly obtain the transverse diameter and anteroposterior diameter of this section (Figure 1). 
Citation: Wu W, Tu Yunhai.Thinks, Chen Y, Shi J (2018) A Study of the impact of Eyelid Opening and Closing on the Volume and Morphology of the Lacrimal Sac. J Eye Dis Disord 3: 116.

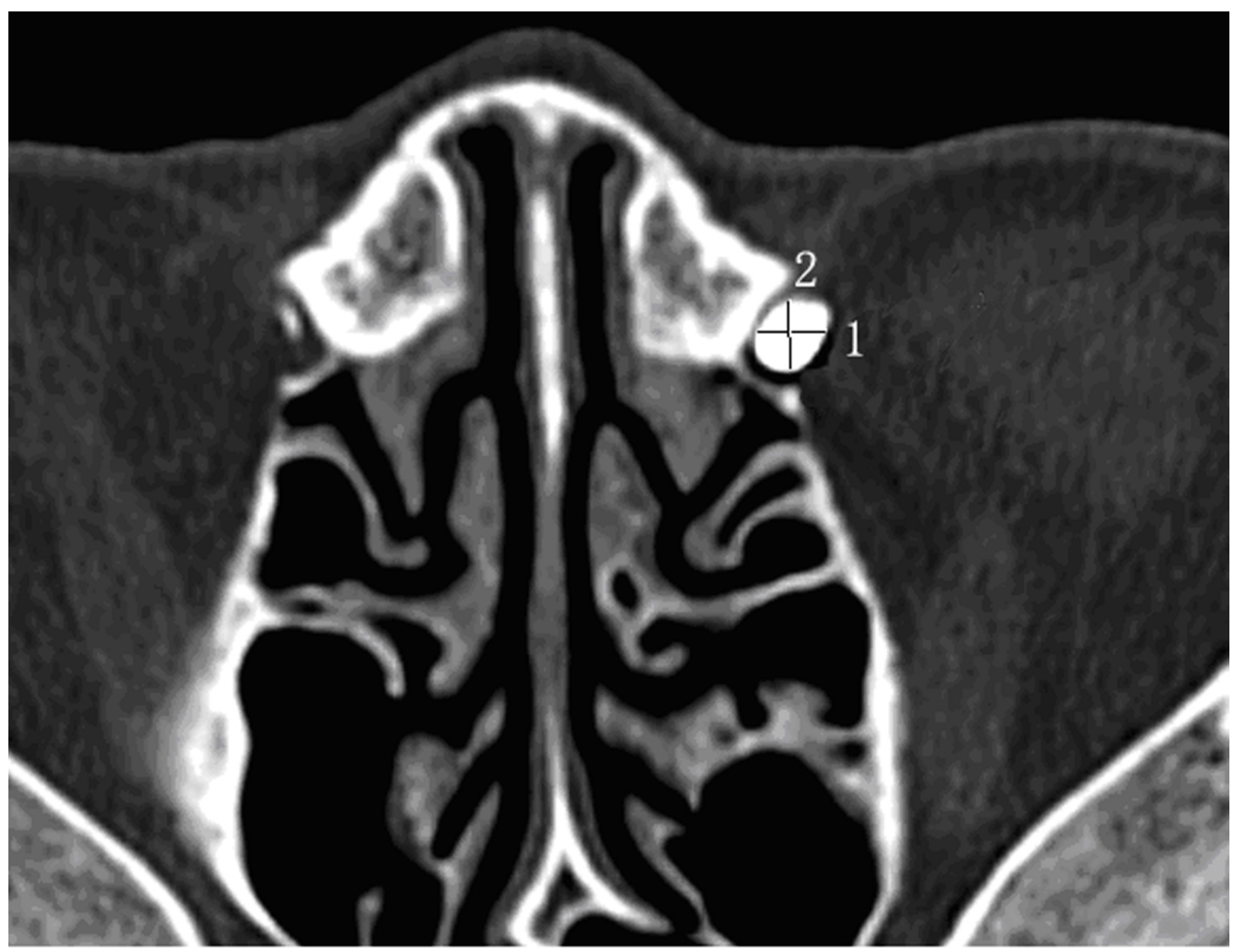

Figure 1. The transverse diameter (1) and anteroposterior diameter (2) of a fixed cross-section could be directly measured using the software that came with the CT system

\section{Results}

This study collected information from a total of 14 patients with chronic dacryocystitis (13 women and one man), with a total of 14 abnormal eyes (six right eyes and eight left eyes). The patients' average age was $41 \pm 13$ years (21-70 years). The CT scanning process went smoothly. When the eyelid was open or closed, the lacrimal sac volume was $0.165 \pm 0.073 \mathrm{~cm}^{2}$ and $0.165 \pm 0.076 \mathrm{~cm}^{2}$, respectively; the difference between the two volumes was not statistically significant. When the eyelid was open, the transverse diameter and anteroposterior diameter at $3 \mathrm{~mm}$ from the top of the lacrimal sac were $0.380 \pm 0.193 \mathrm{~cm}$ and $0.352 \pm 0.144 \mathrm{~cm}$, respectively, and the transverse diameter and anteroposterior diameter at $7 \mathrm{~mm}$ were $0.470 \pm 0.139 \mathrm{~cm}$ and $0.402 \pm 0.132 \mathrm{~cm}$, respectively. When the eyelid was closed, the transverse diameter and anteroposterior diameter at $3 \mathrm{~mm}$ from the top of the lacrimal sac were $0.365 \pm 0.201 \mathrm{~cm}$ and $0.329 \pm 0.154 \mathrm{~cm}$, respectively, and the transverse diameter and anteroposterior diameter at $7 \mathrm{~mm}$ from the top of the lacrimal sac were $0.472 \pm 0.147 \mathrm{~cm}$ and $0.400 \pm 0.128 \mathrm{~cm}$, respectively. When the eyelid was open or closed, except for the anteroposterior diameter at the upper portion of the lacrimal sac, the differences in the transverse diameter and anteroposterior diameter at the two positions of the lacrimal sac were not statistically significant (for details, see Table 1).

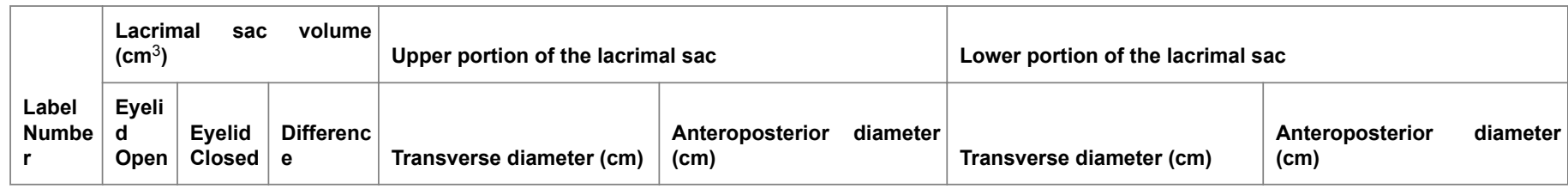




\begin{tabular}{|c|c|c|c|c|c|c|c|c|c|c|c|c|c|c|c|}
\hline & & & & $\begin{array}{l}\text { Eyelid } \\
\text { open }\end{array}$ & $\begin{array}{l}\text { Eyelid } \\
\text { close } \\
\text { d }\end{array}$ & $\begin{array}{l}\text { Differenc } \\
\text { e }\end{array}$ & $\begin{array}{l}\text { Eyeli } \\
d \\
\text { open }\end{array}$ & $\begin{array}{l}\text { Eyelid } \\
\text { closed }\end{array}$ & Difference & $\begin{array}{l}\text { Eyelid } \\
\text { open }\end{array}$ & $\begin{array}{l}\text { Eyelid } \\
\text { closed }\end{array}$ & $\begin{array}{l}\text { Differenc } \\
\text { e }\end{array}$ & $\begin{array}{l}\text { Eyelid } \\
\text { open }\end{array}$ & $\begin{array}{l}\text { Eyelid } \\
\text { closed }\end{array}$ & $\begin{array}{l}\text { Differenc } \\
\text { e }\end{array}$ \\
\hline 1 & 0.12 & 0.13 & -0.01 & 0.45 & 0.33 & 0.12 & 0.48 & 0.37 & 0.11 & 0.62 & 0.54 & 0.1 & 0.51 & 0.44 & 0.07 \\
\hline 2 & 0.15 & 0.16 & -0.01 & 0.34 & 0.39 & -0.05 & 0.32 & 0.29 & 0.03 & 0.53 & 0.58 & -0.1 & 0.36 & 0.37 & 0 \\
\hline 3 & 0.12 & 0.12 & 0 & 0.38 & 0.42 & -0.04 & 0.42 & 0.42 & 0 & 0.55 & 0.59 & 0 & 0.53 & 0.53 & 0 \\
\hline 4 & 0.15 & 0.15 & 0 & 0.85 & 0.88 & -0.03 & 0.72 & 0.78 & -0.1 & 0.36 & 0.37 & 0 & 0.43 & 0.39 & 0.04 \\
\hline 5 & 0.13 & 0.14 & -0.01 & 0.71 & 0.68 & 0.03 & 0.34 & 0.3 & 0.04 & 0.64 & 0.64 & 0 & 0.43 & 0.45 & 0 \\
\hline 6 & 0.37 & 0.39 & -0.02 & 0.49 & 0.45 & 0.04 & 0.47 & 0.41 & 0.06 & 0.71 & 0.75 & 0 & 0.72 & 0.72 & 0 \\
\hline 7 & 0.19 & 0.18 & 0.01 & 0.31 & 0.26 & 0.05 & 0.31 & 0.29 & 0.02 & 0.46 & 0.4 & 0.1 & 0.38 & 0.43 & -0.1 \\
\hline 8 & 0.15 & 0.15 & 0 & 0.3 & 0.31 & -0.01 & 0.35 & 0.34 & 0.01 & 0.42 & 0.41 & 0 & 0.39 & 0.41 & 0 \\
\hline 9 & 0.17 & 0.18 & -0.01 & 0.3 & 0.26 & 0.04 & 0.41 & 0.36 & 0.05 & 0.43 & 0.42 & 0 & 0.46 & 0.45 & 0.01 \\
\hline 10 & 0.05 & 0.04 & 0.01 & 0.17 & 0.15 & 0.02 & 0.23 & 0.19 & 0.04 & 0.19 & 0.16 & 0 & 0.27 & 0.25 & 0.02 \\
\hline 11 & 0.13 & 0.13 & 0 & 0.2 & 0.16 & 0.04 & 0.31 & 0.3 & 0.01 & 0.43 & 0.44 & 0 & 0.28 & 0.27 & 0.01 \\
\hline 12 & 0.15 & 0.15 & 0 & 0.23 & 0.23 & 0 & 0.19 & 0.2 & 0 & 0.31 & 0.35 & 0 & 0.25 & 0.24 & 0.01 \\
\hline 13 & 0.25 & 0.23 & 0.02 & 0.23 & 0.22 & 0.01 & 0.17 & 0.14 & 0.03 & 0.39 & 0.41 & 0 & 0.21 & 0.25 & 0 \\
\hline 14 & 0.18 & 0.17 & 0.01 & 0.36 & 0.37 & -0.01 & 0.21 & 0.22 & 0 & 0.54 & 0.55 & 0 & 0.41 & 0.4 & 0.01 \\
\hline & \multicolumn{2}{|c|}{$P=0.608$} & & $\begin{array}{l}P=0.23 \\
7\end{array}$ & & & \multicolumn{2}{|c|}{$P=0.037$} & & \multicolumn{2}{|l|}{$P=0.693$} & & $P=1$ & & \\
\hline
\end{tabular}

Table 1: The changes of lacrimal volume and morphology parameters when the eyelid was open or closed.

\section{Discussion and Conclusion}

Through CT dacryocystography of patients with nasolacrimal duct obstruction, the present study found that the lacrimal sac volume and the anteroposterior diameter and transverse diameter of the upper and lower portions of the lacrimal sac were not significantly changed when the eyelid was closed, except that the anteroposterior diameter of the upper lacrimal sac became smaller.

The CT dacryocystography technique used in this study is noninvasive and has a high resolution and an ability to provide clear, threedimensional images of the lacrimal sac $[7,8]$. The $\mathrm{B}$ ultrasound scanning used conventionally may press the surrounding tissues and affect the measurement of the lacrimal sac volume. Although radionuclide scintigraphy examination, the digital subtraction technique, and other X-ray examination methods can be used to dynamically monitor dacryocystography, they cannot provide threedimensional imaging results. MRI requires a longer examination time and therefore may not produce an accurate examination of the area when the eyelid is closed or open.

There are several controversies concerning the tear drainage mechanism, but the role of the canaliculus in tear drainage has been accepted by most scholars. Jones [9] conducted studies based on the anatomical structure of the lacrimal duct and found that the horizontal and vertical portions of the canaliculus were surrounded by the orbicularis oculi muscle, resulting in a sphincter-like effect that can maintain the opening and closing of the lumen. When the eyelid is opened, the orbicularis oculi muscle is relaxed, and the canaliculus regresses. As a result, the lacrimal fluid is sucked into the canaliculus through siphon action at the punctum. When the eyelid is closed, the orbicularis oculi muscle contracts, causing the canaliculus to become shorter, prompting the lacrimal fluid to be secreted from the canaliculus into the lacrimal sac. Through high-speed photography of the inner canthus and lacrimal river, Doane et al. [2] confirmed that the canaliculus is shortened when the eyelid is closed. Similarly, many scholars [10-14] have confirmed the role of the canaliculus in tear drainage from physiological and imaging perspectives. Therefore, the role of the lacrimal sac in tear drainage has become the focus of the controversy.

Since the 18th century, scholars have described the orbicularis oculi muscle at the lacrimal sac [15], which is now the Horner muscle, in detail and have proposed a lacrimal pump mechanism: When contracting, the Horner muscle, which is attached to the rear wall of the lacrimal sac, drags the rear wall of the lacrimal sac outward, causing the lacrimal sac to expand and create negative pressure; the lacrimal fluid thus quickly flows into the lacrimal sac. When the muscle stops contracting, the elasticity of the lacrimal wall makes it retract, causing the lacrimal fluid to flow into the nasal cavity. Subsequently, Jones [16] redid an anatomical analysis of the orbicularis oculi muscle, dividing it into three parts (pretarsal muscle, preseptal muscle and orbital muscle), and suggested that closure of the eyelid causes contraction of the pretarsal muscle. As a result, the tarsus is squeezed toward the inside of eyelid, and the canaliculus is shortened by the force, causing excretion of the lacrimal fluid into the lacrimal sac. At the same time, the preseptal muscle contracts, causing the lacrimal sac to create negative pressure, due to which the lacrimal fluid enters the lacrimal sac. When the eyelid is opened, the lacrimal 
sac regresses due to the elasticity of the lacrimal septum, causing the lacrimal fluid to be excreted into the nasal cavity, which is the lacrimal pump theory. The key to this theory is that when the eyelid is closed, the lacrimal sac volume increases, and negative pressure is present. In 1992, while patients were blinking their eyes after dacryocystorhinostomy, Becker [17] used a nasal endoscope to observe and record images of the motion of the lacrimal sac's outer wall. Later, he proposed the "tricompartment model of the lacrimal pump mechanism," which revised and supplemented the theory proposed by Jones. When the eyelid is closed, the orbicularis oculi muscle contracts to compress the canaliculus so that its lumen is closed, and the upper portion of the outer wall of the lacrimal sac moves outward to create negative pressure, causing the lacrimal fluid to enter the lacrimal sac from the canaliculus. Meanwhile, the lower portion of the outer wall of the lacrimal sac drags inward, thereby creating positive pressure in the lower portion of the lacrimal sac and nasolacrimal duct, causing the lacrimal fluid to flow into the nasolacrimal duct, where it is excreted into the nasal cavity. When the eyelid is opened, the orbicularis oculi muscle is relaxed, the canaliculus regresses, and the upper portion of the outer wall of the lacrimal sac moves inward, creating negative pressure in the canaliculus; the lacrimal fluid then flows from the lacrimal lake into the canaliculus, and the inner pressure of the upper portion of the lacrimal sac is increased so that the Rosenmuller valve is closed. At the same time, the lower portion of the outer wall of the lacrimal sac moves outward, thereby creating negative pressure in the inferior lacrimal sac and the nasolacrimal duct, pushing the lacrimal fluid to flow into the lower portion of the lacrimal sac and nasolacrimal duct. Lee et al. [6] performed binocular fluoroscopic dacryocystography in 16 patients with unilateral epiphora who were in a supine position to examine the dynamic changes in the canaliculus and lacrimal sac while the patients were blinking their eyes. The researchers found that the length of the inferior lacrimal duct was shortened and that the width of the upper portion of the lacrimal sac was increased. However, the lower portion of the lacrimal sac did not significantly change. In addition, neither the study of MRI dacryocystography by Amrith et al. [4] nor the present study observed lacrimal sac expansion when the eyelid was closed. Moreover, Pavlidis et al. [6], who used an ultrasound scanning technique, found compression of the lacrimal sac. However, physiological studies showed that the lacrimal sac is under positive pressure when the eyelid is closed $[11,15]$, which does not seem to support the above views.

Lee et al. [5] used a digital subtraction technique to study the impact of eyelid opening and closing on the morphology of the lacrimal sac. Although they studied the changes in the upper and lower portions of the lacrimal sac when the eyelid was opened or closed, they did not provide detailed position information. Orhan et al. [9] found that the lacrimal canalicular orifice was located $2.79 \pm 1.02(0.8-4.9) \mathrm{mm}$ from the dome of the lacrimal sac and $8.56 \pm 1.66(6.3-12.88) \mathrm{mm}$ from the bottom of the lacrimal sac. Therefore, based on the anatomical study by Orhan et al., the present study used the diameter at $3 \mathrm{~mm}$ from the dome of the lacrimal sac to determine the area of the upper portion of the lacrimal sac and the diameter at $7 \mathrm{~mm}$ from the dome to determine the area of the lower portion of the lacrimal sac. Many researchers have reported [18-20] that mucosal folds exist at the canalicular entrance of the lacrimal sac and that these mucosal folds can open and close when the eyelid opens or closes. Using a high-definition video camera, Kakizaki et al. [21] observed the changes in the internal canalicular orifice along with opening and closure of the eyelid after dacryocystorhinostomy. The internal canalicular orifice was closed when the eyelid opened and was open when the eyelid closed. In particular, the internal canalicular orifice opened when the eyelid closed firmly, while the outward movement of the lacrimal sac wall was not obvious. Therefore, the expansion of the upper portion of the lacrimal sac observed by Lee et al. [6] when the eyelid was closed may be related to the opening of the valve of the lacrimal canaliculus. The present study shows that the anteroposterior diameter of the upper portion of the lacrimal sac decreases when the eyelid is closed and that the difference is statistically significant. However, while one patient had a significant change in the anteroposterior diameter, the changes in the anteroposterior diameter in the other patients were not significant. Previously, Theodossiadis et al. [22] used a radionuclide scintigraphy technique in their study and found that the size of the lacrimal sac decreased when the eyelid was closed. However, the imaging resolution of this technique is relatively low, and there were certain errors in the study.

In summary, we suggest that the volume and morphology of the lacrimal sac do not significantly change during the opening and closing of the eyelid and that the effect of the pressure change in the lacrimal duct during tear drainage is limited. The detailed role of the lacrimal sac needs further study. Limitations of the present study include the relatively small sample size and the lack of supportive physiological data. The orbital CT scan is also slow and cannot record dynamically changing data; however, in the present study, we repeatedly told the patients to keep their eyes closed firmly when the eyelid needed to be closed to ensure that the orbicularis oculi muscle was contracted. Additionally, the present study used patients with nasolacrimal duct obstruction, who may present differences compared to healthy subjects, as study subjects. However, in the patients with nasolacrimal duct obstruction, a loss of contrast agent through the nasal cavity did not occur and therefore likely did not affect the experimental results when the eyelid opened and closed.

\section{Funding}

This work is supported by the funds of Wenzhou Municipal Science and Technology Bureau (Y20160057).

\section{References}

1. Jones LT (1973) Anatomy of the tear system. Int Ophthalmol Clin 13: 3-22.

2. Doane MG (1981) Blinking and the mechanics of the lacrimal drainage system. Ophthalmology 88: 844-851.

3. Becker BB (1992) Tricompartment model of the lacrimal pump mechanism. Ophthalmology 99: 1139-1145.

4. Amrith S, Goh PS, Wang SC (2005) Tear flow dynamics in the human nasolacrimal ducts pilot study using dynamic magnetic resonance imaging. Graefes Arch Clin Exp Ophthalmol 243: 127-131.

5. Lee MJ, Kyung HS, Han MH, Choung HK, Kim NJ, et al. (2011) Evaluation of lacrimal tear drainage mechanism using dynamic fluoroscopic dacryocystography. Ophthal Plast Reconstr Surg 27: 164-167.

6. Pavlidis M, Stupp T, Grenzebach U, Busse H, Thanos S (2005) Ultrasonic visualization of the effect of blinking on the lacrimal pump mechanism. Graefes Arch Clin Exp Ophthalmol 243: 228-234.

7. Rached PA, Canola JC, Schluter C (2011) Computed tomographicdacryocystography (CT-DCG) of the normal canine nasolacrimal drainage system with three-dimensional reconstruction. Vet Ophthalmol 14: 174-9.

8. Nykamp SG, Scrivani PV, Pease AP (2004) Computed tomography dacryocystography evaluation of the nasolacrimal apparatus. Vet Radiol Ultrasound 45: 23-28. 
Citation: Wu W, Tu Yunhai.Thinks, Chen Y, Shi J (2018) A Study of the impact of Eyelid Opening and Closing on the Volume and Morphology of the Lacrimal Sac. J Eye Dis Disord 3: 116.

Page 6 of 6

9. Orhan M, Govsa F, Saylam C (2009) Anatomical details used in the surgical reconstruction of the lacrimal canaliculus: cadaveric study. Surg Radiol Anat 31: 745-753.

10. Rosengren B (1972) On lacrimal drainage Ophthalmologica 164: 409-421.

11. Hill JC, Bethell W, Smirmaul HJ (1974) Lacrimal drainage--a dynamic evaluation. Part I--mechanics of tear transport. Can J Ophthalmol 9: 411-416.

12. Hill JC, Bethell W, Smirmaul HJ (1974) Lacrimal drainage--a dynamic evaluation. Part II--clinical aspects. Can J Ophthalmol 9417-9424.

13. Wilson G, Merrill R (1976) The lacrimal drainage system: pressure changes in the canaliculus. Am J Optom Physiol Opt 53: 55-59.

14. Chavis RM, Welham RA, Maisey MN (1978) Quantitative lacrimal scintillography. Arch Ophthalmol 96: 2066-2068.

15. Reifler DM (1996) Early descriptions of Horner's muscle and the lacrimal pump. Surv Ophthalmol 41: 127-134.

16. JONES LT (1957) Epiphora. II. Its relation to the anatomic structures and surgery of the medial canthal region. Am J Ophthalmol 43: 203-212.
17. Becker BB (1992) Tricompartment model of the lacrimal pump mechanism. Ophthalmology 99: 1139-1145.

18. You Y, Cao J, Zhang X (2016) In Vivo and Cadaver Studies of the Canalicular/Lacrimal Sac Mucosal Folds. J Ophthalmol: 3453908.

19. Zoumalan CI, Joseph JM, Lelli GJ Jr, Segal KL, Adeleye A, et al. (2011) Evaluation of the canalicular entrance into the lacrimal sac: an anatomical study. Ophthal Plast Reconstr Surg 27: 298-303.

20. Aubaret E (1908) The valves of the lacrymo-nasal passages. Arch Ophthalmol 28: 211-236

21. Kakizaki H, Takahashi Y, Miyazaki H, Nakamura Y (2013) Movement of internal canalicular orifice in association with blinking: direct observation after dacryocystorhinostomy. Am J Ophthalmol 156: 1051-1055.

22. Theodossiadis G, Panopoulos M, Chatzoulis D, Koutoulidis K, Samaras B, et al. (1979) How do tears drain? Technetium studies. Can J Ophthalmol 14: $169-172$. 